\title{
InSPIREd - Advances in Conformal Printing: 3D Printing onto Unknown Uneven Surfaces ***
}

\author{
Nils Bausch, David P. Dawkins and Regina Frei ${ }^{1}$
}

\begin{abstract}
The term Conformal Printing refers to 3D printing onto uneven surfaces. Whilst some very high priced solutions exist for known uneven surfaces, where the toolpath is generated in advance, based on CAD data of the object to be printed onto, there is nothing available yet for conformal printing onto unknown uneven surfaces that are scanned on-the-fly. A low-cost prototype named 'InSPIREd' was recently developed to achieve this, made possible with a combination of multidisciplinary expertise, ingenuity and problem tackling knowhow, and the latest improvements are presented in this paper. The prototype now includes more capable scanning technology and a simplified calibration procedure.
\end{abstract}

\section{INTRODUCTION}

3D printing usually proceeds in horizontal layers deposited onto a flat substrate. The exception are very expensive machines for printing antenna onto electronic boards. However, these printers have three limitations: first, the substrate shape needs to be known in advance to generate the toolpath; second, the printed elements usually only consist of one layer (although deposited onto a 3D object); and third, these printers cost up to half a million GBP.

The prototype developed for conformal printing onto unknown uneven surfaces addresses all three limitations: it is low cost (hardware and software components for a total of under GBP 2000); it can print onto unknown 3D objects, scanning them and generating the toolpath on the fly; and it can print 3D objects composed of many stacked layers. InSPIREd stands for 'Integrated Seven-axis Printer / scanner Reactive SurfacE deposition'.

A first version of the prototype being presented in [1], this paper introduces an improved version of InSPIREd, where the laser line sweep was replaced by a projector and lens to adjust for close throw distance, and a simplified calibration procedure. The calibration panel can now be removed after initial calibration, as long as projector, lens and camera do not change position. This assumption should be fine due to additional fixtures for this equipment. Furthermore, improved and new pictures taken from Rhino provide better explanations.

Figure 1 depicts the outcome of the Prusa i3's conversion to a 7 DOF printer.

* This work was supported by the University of Portsmouth - Research Development Framework (RDF) 2015.

** Our sincere thanks go to the DC42 firmware developer David Crocker from the RepRap community.

${ }^{1}$ All authors are with the School of Engineering, University of Portsmouth, UK. workenilsbausch.net, david.dawkins@myport.ac.uk,

work@reginafrei.ch

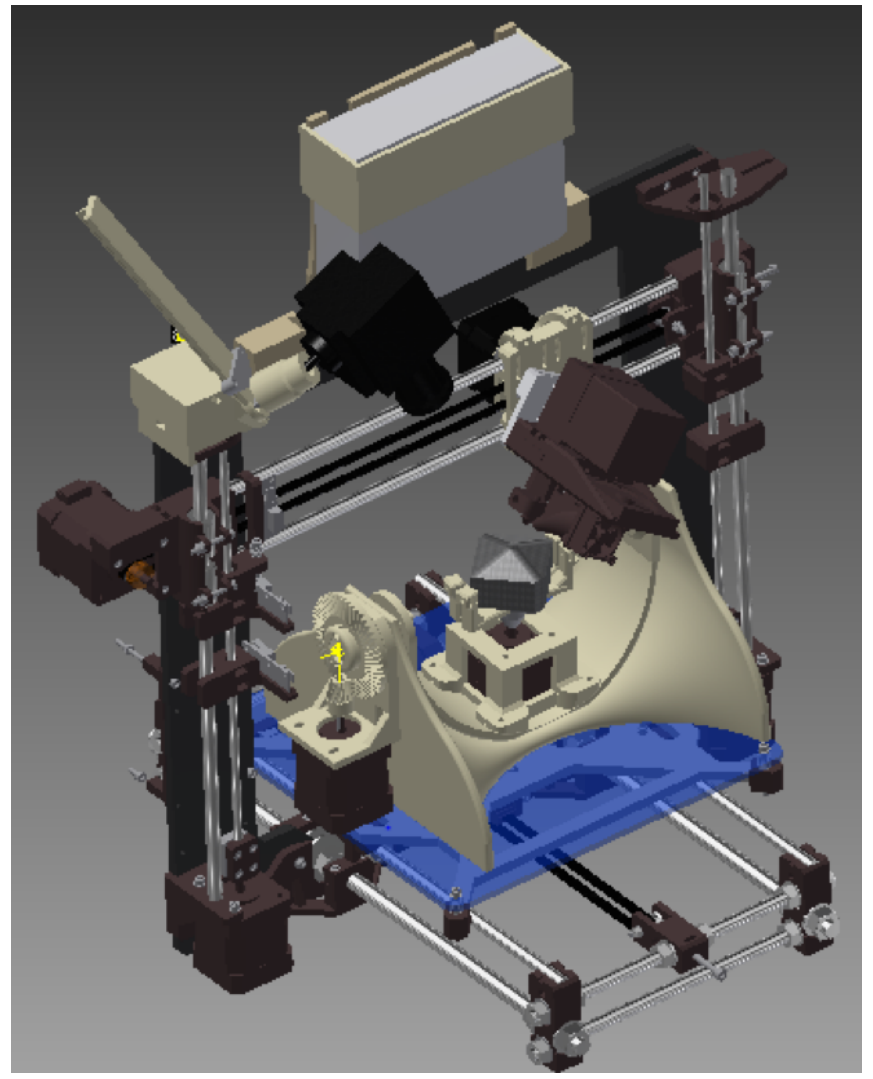

Fig. 1. The improved InSPIREd prototype

\section{RELATED WORK}

Conformal printing: It appears that there is currently no solution for printing onto unknown uneven surfaces or 3D objects, at any scale. The only commercially available solutions for printing onto known uneven surfaces - aerosol jet printers [2] - are made for printing electronic circuits and antennae [3] and requires inks to be transformed into aerosols. [4] modified an industrial FDM system to increase its degrees of freedom, allowing the machine to perform freeform manufacturing including complex curved shapes and deposition orientations other than horizontal. The system is also able to print onto existing parts. However, the part surface needs to be known to generate the toolpath.

Low cost $3 D$ printers with more degrees of freedom:

[5] reports on a 3D printer with $6 \mathrm{DOF}$, whereof 3 are in the base plate. The plate can be angled, such that the object being printed can be more upright and does not need the support structures otherwise required. However, the position 
of the print head is not adjusted and will hence not remain orthogonal to the surface being deposited. This is something the 5AXISMAKER (http://www. 5 axismaker.com/) can do; it is a multi-functional robotic platform that can adopt additive or subtractive tools for various fabrication needs.

$3 D$ scanning: [6] surveyed 3D scanning technology for small objects, differing between rigid and non-rigid registration. Gathered data can be represented as point clouds or meshes composed of usually triangular shapes of varying size, angle and orientation. The DAVID laser scanner [7], [8] uses structured light triangulation (SLS). In principle, this method can use a single laser line, video camera, the object, and behind it a calibration panel as a reference geometry. Multiple sweeps of the laser line across the object will reflect light to the camera. The scanned object's surface geometry can then be calculated [9], which is carried out by the DAVID4 software package. An advanced version of this replaces the laser with a projector, which projects a line pattern at close distance onto the object.

Point cloud data and mesh conversion: The output of a 3D scan, or a CAD system for CAM/CNC applications is usually a mesh or point cloud. From this data, a toolpath is generated for the actual machining. [10] presented a method for toolpath generation based on triangular meshes and the proposed method can be used to augment and automate freeform surface machining. [11] proposed a simple algorithm to generate a multi-axis toolpath for machining. This direct method for constructing a 3D triangular mesh from the point cloud data created mesh points based on where the machining tool would contact the object.

\section{STRATEGY}

An ideal workflow to arrive at the desired outcome of 3D printing an object onto a previously unknown surface (object) can be broken down into the following steps:

1) Place and fasten an unknown object into a $3 D$ printer

2) Scan the unknown object and extract the data

3) Superimpose the existing object with the new object

4) Generate the toolpaths for the $3 \mathrm{D}$ printer

5) Print the new object

An overview of the approach taken for each step follows; the detailed implementation is described in [1].

Placing the object: An unknown object could be of any form and shape and therefore restrictions are necessary, to conform with the available overall space of the machine as well as the necessary printing space for the new object. The unknown object should be mounted appropriately to reduce further human intervention and minimise disturbance.

Scanning the object: The unknown object will be scanned inside the $3 \mathrm{D}$ printer and if necessary rotated to capture all the surfaces that will be printed on or to improve the scanning procedure. Depending on the scanning method, accuracy and elapsed time can vary. The current method can scan a variety of different shapes. To exemplify the working principle, a generic hemispherical structure was used. The scanning or printing onto shadowed parts of a structure created by protruding or overlapping features is currently not supported.
Superimposing object data: The previously unknown object data is then superimposed with an existing design for an object that is to be 3D printed. This can also be described as a 'boolean' operation, whereby areas of intersection will form the boundaries from where parts of the new object remain or are deleted accordingly. This step requires a bespoke software system to superimpose the objects.

Generating toolpath data and printing: A bespoke software system is then used to generate the necessary toolpath data required to control the $3 \mathrm{D}$ printer. For reasons of simplicity, only printing on top of an object was considered.

\section{SETUP AND DEVELOPMENT}

Amongst a selection of commercially available systems, the Prusa RepRap i3 was chosen for several reasons: to shorten the process of building the prototype, improve its ease of use, keep costs low and to benefit from the possibility to modify pre-existing firmware. The printer was converted to include seven Degrees Of Freedom (DOF) for FDM. The DOF include linear movements along the $X, Y$, and $Z$ axes as well as angular movements around these in $\alpha, \beta$, and $\gamma$ direction and the extruder output.

\section{A. Axes and DOF}

The conversion to a 7 DOF machine was realised by creating new and adjusting existing parts of the printer. Initially, the new parts where designed using FDM rapid prototyping methods to reduce the time from design to manufacturing. Later the parts were replaced by aluminium parts to increase stability and rigidity.

The machine layout was adjusted to house another three stepper motors for the additional DOF. Although the prototype 3D printer has 6 DOF available in the current implementation (the 7th being material extrusion), it only supports 5 concurrent DOF at this stage: one of the rotary axes must remain in its home position to ensure a perpendicular orientation of the print head to the object surface. For details see [1].

\section{B. $3 D$ scanner and GUI}

In the first version of the prototype, the 3D scanner consisted of a blue line laser and a monochrome camera. The improved version of the prototype now uses a Digital Light Processing (DLP) projector instead, which projects a pattern onto an object, that is then captured by the camera and processed by the DAVID4 software. A focussing lens was added to the DLP projector to enable the short throwing distance needed for a compact design. A further benefit of the new approach is the one-time calibration with a calibration panel that had to be performed at the start. Only a change in position of the DLP projector, lens or camera would require a recalibration, but not the exchange of an object. The improvements in comparison to the line-laser are: higher scanning accuracy (see Figure 13); shortened process time due to exclusion of line-laser sweeps and recalibration when exchanging objects. 
A host controller software Graphical User Interface (GUI) was developed in $\mathrm{C \#}$ to simplify setup and communication with the $3 \mathrm{D}$ printer.

\section{Placing the object and calibration}

Due to the underlying technique of structured light triangulation, a removable calibration panel was incorporated into the printer design. This panel no longer needs to remain in place throughout the scanning procedure. The main advantage of using this type of 3D scanner and software is the possibility to reproduce the surface geometry quickly and to a high standard precision and repeatability, $\pm 0.02 \mathrm{~mm}$. After initial calibration, the user places an object into the printer's gripper and secures it by fastening the bolts.

\section{Scanning}

Once the scanner is calibrated and the object is placed, the user can initiate the scanning procedure by using the semiautomatic custom GUI host program to connect, scan, manipulate, and send toolpaths. After each scanning procedure, the user can visually check for satisfactory surface geometry recorded by the DAVID4 software and repeat the procedure if more / better surface data is needed. The DAVID4 software fuses these different scans into one coherent object. The user must then ensure no irrelevant artefacts have been captured. This is done by visual inspection of the scanned object within the DAVID4 software. Wrongly included artefacts are selected and the points representing them removed.

The point cloud data obtained from the scanning is smoothed and averaged during this process using DAVID4 software, which is necessary to account for reflected scattered light from the object surface. The aperture setting on the camera may need to be adjusted to suit the ambient light conditions; similarly the absorption, reflectivity and emissivity of the object being scanned affect the camera's ability to pick up the laser light.

Moreover, the printer and scanner has limitations to the angles that can be scanned. Another limitation is the print head size, which hinders approaches to concave corners with sharp angles.

After capturing the surface geometry, the user exports the file as a high density STereoLithography (STL) mesh file, with facets selected in DAVID4 no greater than $0.5 \mathrm{~mm}$.

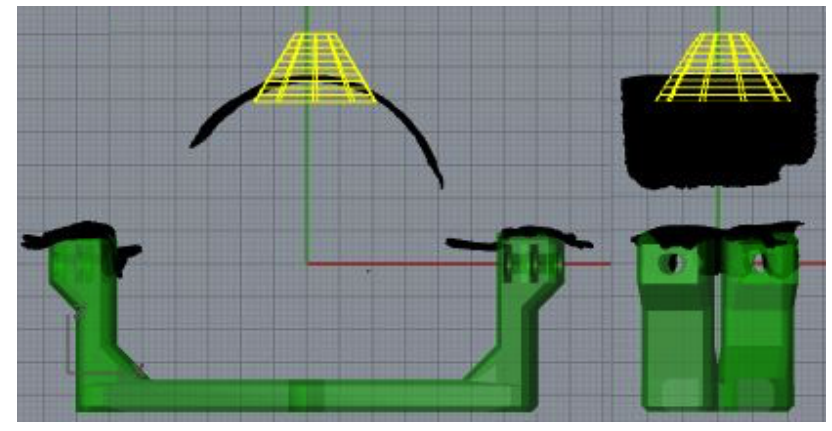

Fig. 2. Alignment of the green reference gripper with the black scanned object and the yellow cone-shaped surface mesh of the object to-be printed viewed from the $Z X$ and $Z Y$ plane.

\section{E. Superimposing object data}

The superposition (or positioning of the object to-be printed with reference to the scanned object) is carried out in Rhino / Grasshopper, allowing for easy manipulation and control. The main function of Rhino / Grasshopper is to slice the to-be printed object and obtain toolpath coordinates, including facets and vertices normals, lengths and volume, as a part of the object surface geometry. The overall outcome is a toolpath consisting of positional coordinates, length of the line segment, and the vertices' normal coordinates, and from this the volumetric extrusion can be computed.

By importing both the object to-be printed and the scanned object into Rhino as STL files, one can manipulate the scanned object to match up with the printer's grippers, which are used as an alignment (see Figure 2). Importing the gripped object into the workflow of Rhino allows for visual inspection and alignment, where the home position is unified between the software and printer. The toolpath is generated relative to these coordinates.

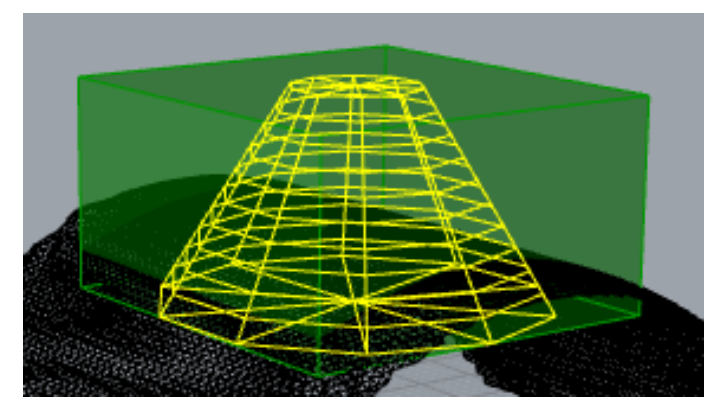

Fig. 3. Bounding box, i.e. maximum extents of object to be printed

When the scanned object is in position, the user needs to define a suitable location for the object to-be printed, such that it intersects the scanned object. Before a boolean intersection can be performed, two volumetric objects are created: the 'new' object's envelope forms the first object, and a combined stack of layers from the scanned object's surface forms the second object. A bounding box, as illustrated in Figure 3 enveloping the 'new' object is created to capture height, width, and depth. The number of layers in the stack depends on the printed layer height, which depends on the 3D printer setup, and the 'new' object's bounding box as limits. Once the layer stack, now representing a volume, is created, a boolean intersection is performed, whereby the common parts of both objects are kept and results, in this case, in a sliced mesh that conforms to the object's surface and forms the new base of the object to-be printed. This method can currently only operate with scanned surfaces that have no holes in the area of intersection with the to-be printed object, which was visually verified by the user.

Figure 4 shows in green the result of a procedure to define the surface for the scanned object's data, which was so far only a point cloud. This step was necessary to facilitate the creation of an object for the boolean volumetric operation.

Lofting / stacking the layers, illustrated in Figure 5, is specific to the printer we are using (each about $1 \mathrm{~mm}$ thick 


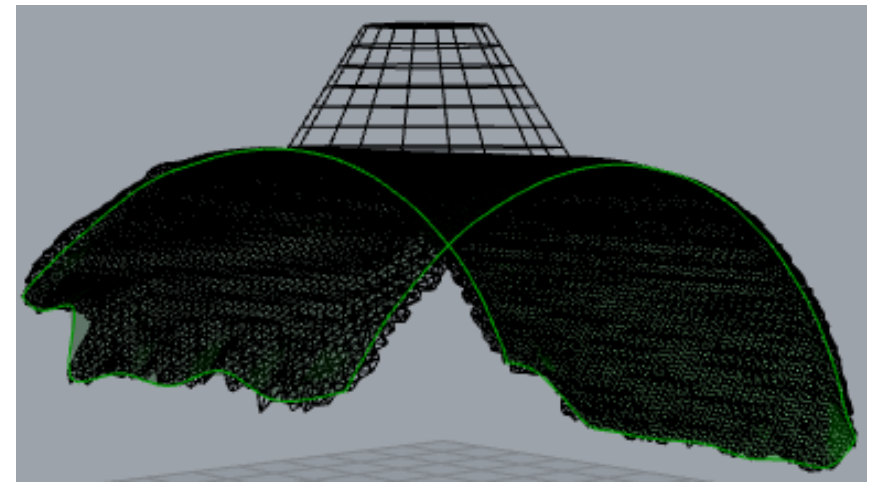

Fig. 4. Defining the surface layer

in simulation for visualisation); in reality, these layers are as thin as the printer can do. The top end of the stack is given by bounding box (Figure 3). The layering of the surface on top of each other created a new volumetric object.

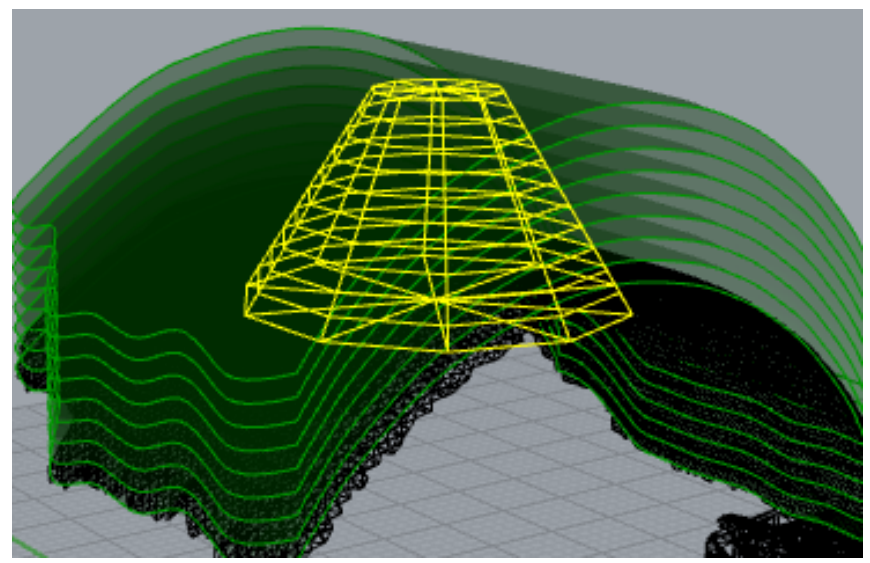

Fig. 5. Array of layers

At a next step, a volumetric boolean 'AND' operation was then applied to the new to-be-printed object and the volume consisting of the stacked surface. Figure 6 represents the superimposition, showing what needs to be printed in each step from the intersection of layers with the object (no toolpath yet, only graphical information at this stage). The output of the boolean operation clearly shows the outline of the to-be-printed object and inside of it the stack of layers, that will be printed.

The creation of toolpaths began with the definition of edges for each layer. Each layer consisted of a mesh of points, which are interconnected. To find the edges (see Figure 7), we search for points that have degrees of freedom (i.e. they are not fully constrained). Points somewhere inside the layers are all connected; those on the outside have degrees of freedom, hence they are edges.

\section{F. Generating the toolpath}

The toolpath for the new object consisted of the edges and infill. The edges were found through their DOF and the infill choice is based on the object that is printed. Figures 8

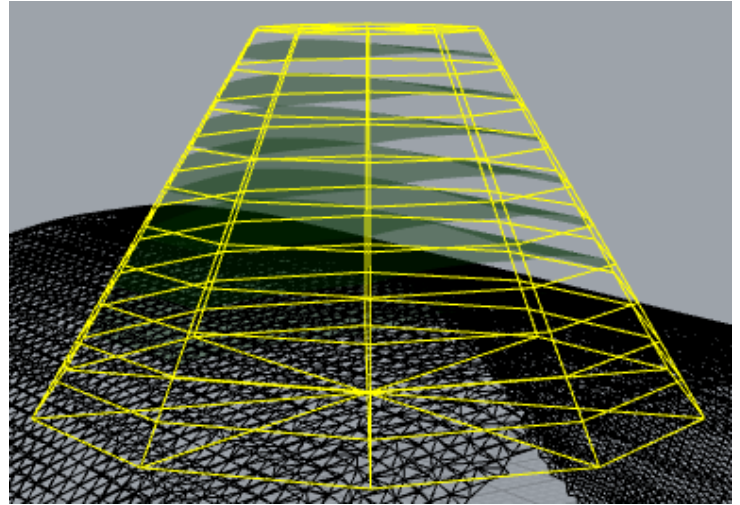

Fig. 6. Superimposition: Boolean AND operation

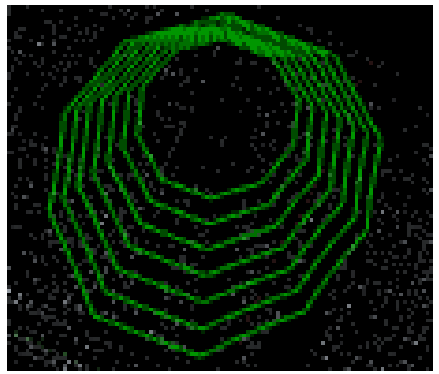

Fig. 7. Finding the edges

and 9 show the use of layers to create an infill (spaced $1 \mathrm{~mm}$ apart) in $X$ and $Y$ direction (the pictures showing only one direction).

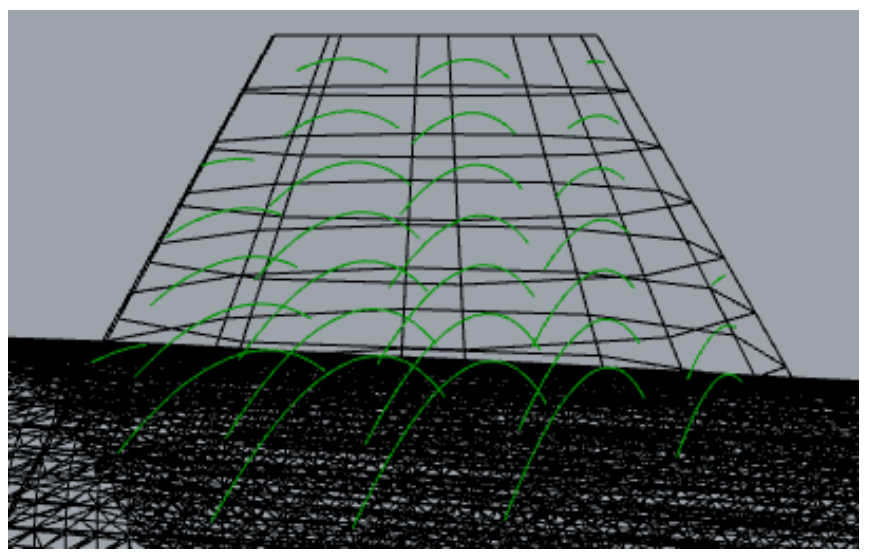

Fig. 8. Infill 1

In a normal 3D printer, the infill and edges are enough to create the toolpath from, but for conformal printing we need more information to correctly control the printhead. Figure 10 shows what we call iso-curves: the layers are not flat, hence the printhead must adjust its path to avoid hitting the object. Therefore, toolpath generation needs to take this into consideration, making iso-hops for adjustments. Usually a 3D printer's printhead moves to the next point without having to respect previously printed parts, as layers are usually stacked in $Z$-axis direction. But due to the nature 


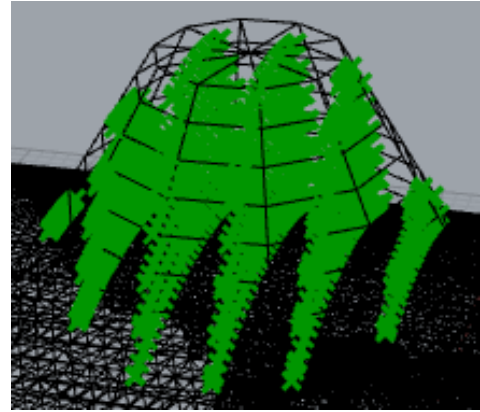

Fig. 9. Infill 2

of the layers conforming to the scanned object's surface, the printhead needs to move in a similar fashion to avoid hitting the object during the process.

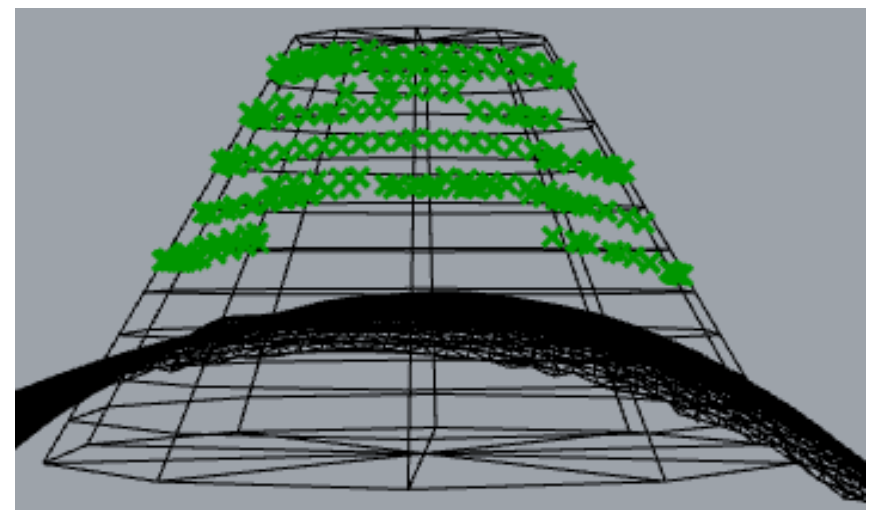

Fig. 10. Iso-curves

The remaining components contain the data parameters of the intersection line's geometry and position (including length, extrusion width and layer height to calculate extrusion volume) and additional infill data was generated as support. Distinct differences to traditional 3D printing were addressed in intermediate steps. At first, the normal vectors of each point to be printed are calculated to ensure perpendicular material deposition. Then a print manoeuvre toolpath is created. In a last step, polylines consisting of closest points combine print and non-print manoeuvres are smoothed by distributing points with a minimal move distance to eliminate jagged print head movements. A simulated 3D printer output can be seen in Figure 11.

\section{G. Printing}

A second bespoke software program in Python was created to generate the toolpath data required to control the $3 \mathrm{D}$ printer. This program converts the exported files from Rhino / Grasshopper into a usable form for 3D printing and the GUI program uploads the resulting G-code to the 3D printer. This step translates the generated toolpath's coordinates into the one used by the 3D printer. [1] provides a detailed explanation of this procedure.

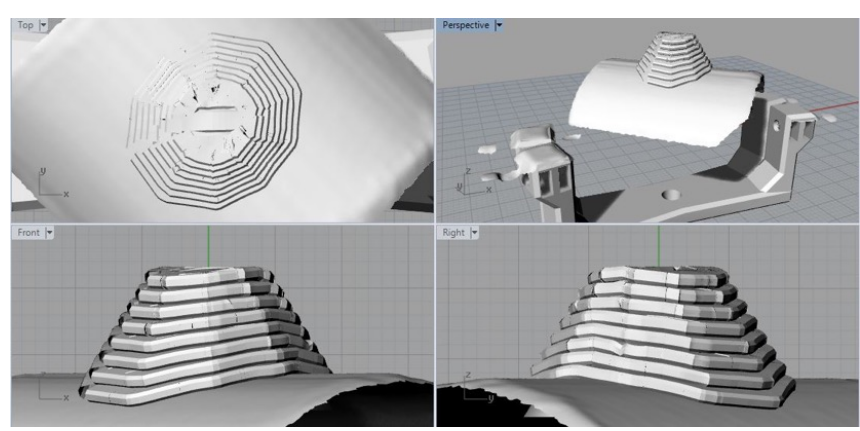

Fig. 11. Toolpath oversized: showing the layers with the selected layer width (adjust to printer capability)

\section{RESULTS AND ASPECTS OF IMPLEMENTATION}

Figure 12 shows the first experimental result of the conetype object being printed on top of an existing round object. The irregularities are down to the printer needing some further fine-tuning. However, the proof-of-concept has been successfully delivered.

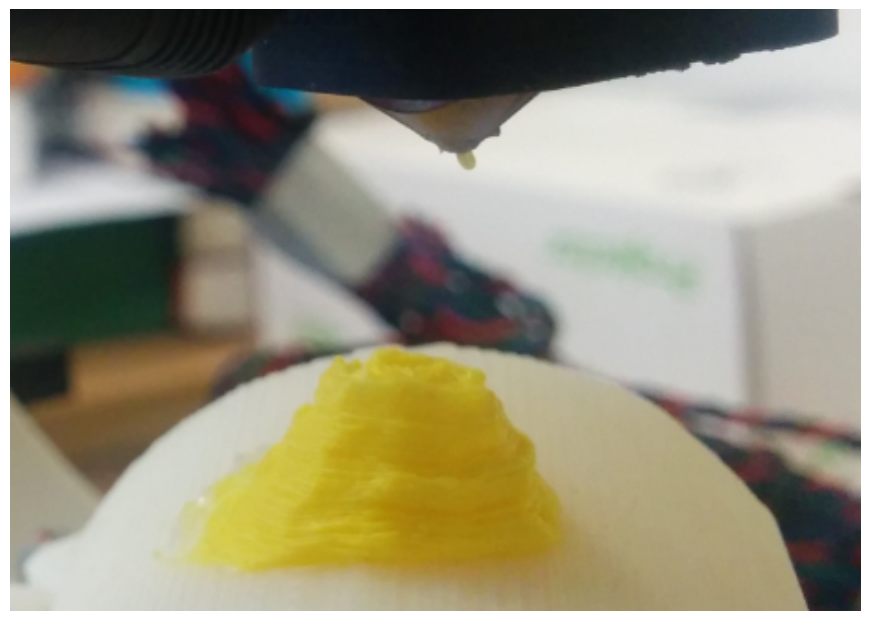

Fig. 12. First printed result

Subsequently, implementation issues are described and current / future improvements explained where necessary, using subsection lettering and headings from Section IV.

B. 3D scanner and GUI: The 3D scanning camera was mounted onto the frame and a mounting platform created to hold the DLP projector in place. Initial tests were performed, and it tuned out that the minimum throw distance of the projector was too large for the setup. Different lenses were tested to focus the projector at an even closer distance.

$C \&$ D.Placing the object, calibration and scanning: The calibration panel for the $3 \mathrm{D}$ scan was redesigned for easy removal and mounting for the scan procedure. The DAVID4 software uses a patterned geometry to correctly determine the size of the scanned object and must be precisely arranged at a $90^{\circ}$ angle to determine the correct object surface distances. As the calibration pattern is not in the centre, it allowed space for a small cutaway enabling rotation about the $Z$ axis, $\alpha$, but only if the gripped object is small enough. Figure 13 shows 
a scan of a printed object held by the prototype grippers.

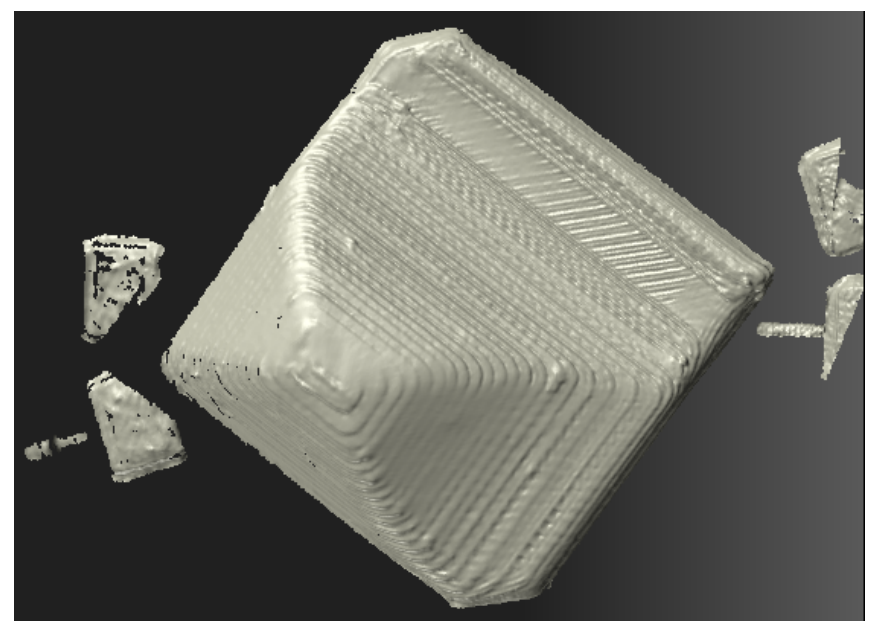

Fig. 13. Scan of a (printed) object with grippers left and right of it

E. Superimposing object data: In the future, an algorithm could be developed to automate the alignment of scanned part with gripper in a Grasshoppers workflow: the rotation tools and detection of the grippers shape could be linked together in such a way that the scanned object gets placed using a 'best fit' method, with minimal user interaction. In comparison to aligning the scanned part with the reference gripper, the orientation and positioning of the object to-be printed is easy to execute. The perimeter, that is edge of the intersection, is currently generated by specifying each point on the toolpath from vertices of the mesh line. Therefore high density meshes have to be used due to the nature of operation. The overall results were accurate.

$F \& G$. Generating toolpath and printing: Once the toolpath perimeter has been generated, the print head comes within printing distance of the object, which is $0.2 \pm 0.04 \mathrm{~mm}$. Printing extensive lengths with a single Gcode command whilst doing rotary movements does currently not lead to ideal results. If the movement was to follow a straight line whilst rotating around the $\beta$ axis, the print head nozzle could dip below or raise above the selected line. This led to the design decision to use very small increments between toolpath points, which in turn prevents the nozzle from crashing into the object or going off path. The firmware could be modified to automatically generate the offsets for the print head if a specific G-code command was to be used. This way the print head tip would always remain at the specified Cartesian coordinate. Also in the future, after calibration of the printer, the print head should follow a 'keep out' boundary box during the movements from its home position to the start of toolpath which would prevent object collisions.

\section{CONCLUSION}

Whilst conformal printing onto previously unknown objects is theoretically feasible because all required technologies are available, it is a challenge to compose an affordable system that combines all involved elements. This paper reports on the progress made in an ongoing project aimed at creating a desk-top printer with all required capabilities. The InSPIREd prototype is able to scan an unknown object to gather point cloud data, superimpose the data with the object to be created on top, generate a toolpath, and print the new object onto the existing object. Currently, the project team is working on technical refinements. Future work will include filling in the currently produced perimeter, much in the same way of conventional slicing, using iso-curves, the utilisation of a SLS projector scanner, and the inclusion of Euclidean / Tait-Bryan rotational angle calculations to utilise all 6 DOF.

To summarise the achievements so far: A hand-full of known technologies were combined to create a prototype with new capabilities. A new methodology was designed in Grasshopper / Rhino3D. All in all, hardware and software cost below GBP 2000, and the InSPIREd prototype is printing successfully. The limitations include that the calibration panel still needs to be used, that there is some manual interaction necessary, and the surface adhesion between the printed objects and the carrier objects has not been studied yet. Ongoing and future work will test other surface shapes and other object shapes, as well as make the printing procedure semi-automatic, with additional programming for the interactions between printer control and Rhino.

\section{REFERENCES}

[1] N. Bausch, D. Dawkins, R. Frei, and S. Klein, "3D printing onto unknown uneven surfaces," in IFAC Mechatronics, ser. IFACPapersOnLine, 2016, vol. 49, no. 21, pp. 583-590.

[2] J. Paulsen, M. Renn, K. Christenson, and R. Plourde, "Printing conformal electronics on 3d structures with aerosol jet technology," in IEEE Future of Instrumentation International Workshop (FIIW), 2012, 2012, pp. 1-4.

[3] J. Adams, E. Duoss, T. Malkowski, M. Motala, B. Ahn, R. Nuzzo, J. Bernhard, and J. Lewis, "Conformal printing of electrically small antennas on three-dimensional surfaces," Advanced Materials, vol. 23, no. 11 , pp. 1335-1340, 2011.

[4] J. Choi, F. Medina, C. Kim, D. Espalin, D. Rodriguez, B. Stucker, and R. Wicker, "Development of a mobile fused deposition modeling system with enhanced manufacturing flexibility," Journal of materials processing technology, vol. 211, no. 3, pp. 424-432, 2011.

[5] H. Hansen, "3d-drucker mit beweglicher druckplatte (3D printer with mobile printing plate)," 2017. [Online]. Available: https: //heise.de/-3594506

[6] G. Tam, Z. Cheng, Y. Lai, F. Langbein, Y. Liu, D. Marshall, R. Martin, X. Sun, and P. Rosin, "Registration of $3 \mathrm{~d}$ point clouds and meshes: a survey from rigid to nonrigid," IEEE Transactions on Visualization and Computer Graphics, vol. 19, no. 7, pp. 1199-1217, 2013.

[7] S. Winkelbach, S. Molkenstruck, and F. M. Wahl, "Low-cost laser range scanner and fast surface registration approach," in Pattern Recognition. Springer, 2006, pp. 718-728.

[8] DAVID, "SLS-2," 2015. [Online]. Available: http://www.david-3d. com/en/products/sls-2

[9] U. Aydar, O. Akyol, and Z. Duran, "A low-cost laser scanning system design," in XXIIIth International CIPA Symposium, Prague, 2011.

[10] T. Chen and Z. Shi, "A tool path generation strategy for three-axis ballend milling of free-form surfaces," Journal of Materials Processing Technology, vol. 208, no. 1-3, pp. $259-263,2008$.

[11] K. Chui, W. Chiu, and K. Yu, "Direct 5-axis toolpath generation from point cloud input using 3d biarc fitting," Robotics and ComputerIntegrated Manufacturing, vol. 24, no. 2, pp. 270 - 286, 2008. 